\title{
Corela
}

Cognition, représentation, langage

$12-2 \mid 2014$

Vol. $12, n^{\circ} 2$

\section{Processus de la traduction : charge cognitive du traducteur}

Esmaeel Farnoud

\section{(2) OpenEdition}

9 Journals

Édition électronique

URL : https://journals.openedition.org/corela/3615

DOI : $10.4000 /$ corela.3615

ISSN : 1638-573X

Éditeur

Cercle linguistique du Centre et de l'Ouest - CerLICO

\section{Référence électronique}

Esmaeel Farnoud, « Processus de la traduction : charge cognitive du traducteur », Corela [En ligne], 12-2 | 2014, mis en ligne le 15 décembre 2014, consulté le 21 septembre 2021. URL : http:// journals.openedition.org/corela/3615; DOI : https://doi.org/10.4000/corela.3615

Ce document a été généré automatiquement le 21 septembre 2021.

\section{(c) (i) (2)(2)}

Corela - cognition, représentation, langage est mis à disposition selon les termes de la licence Creative Commons Attribution - Pas d'Utilisation Commerciale - Partage dans les Mêmes Conditions 4.0 International. 


\title{
Processus de la traduction : charge cognitive du traducteur
}

\author{
Esmaeel Farnoud
}

\section{Introduction}

1 La traduction est un processus de communication qui met en présence au moins 3 personnes :

- La personne (P1) qui énonce dans la langue de départ (LD) ;

- La personne (P2) qui souhaite comprendre le message émis par P1, mais qui ne maîtrise pas (suffisamment) la langue LD; elle a, par contre, l'habitude de communiquer dans la langue d'arrivée (LA);

- Entre les deux se trouve, comme médiateur, le traducteur qui capte les énoncés émis par P1 en LD afin de communiquer leur contenu en LA à P2.

2 Voici le schéma triangulaire du processus traduisant:

Figure 1

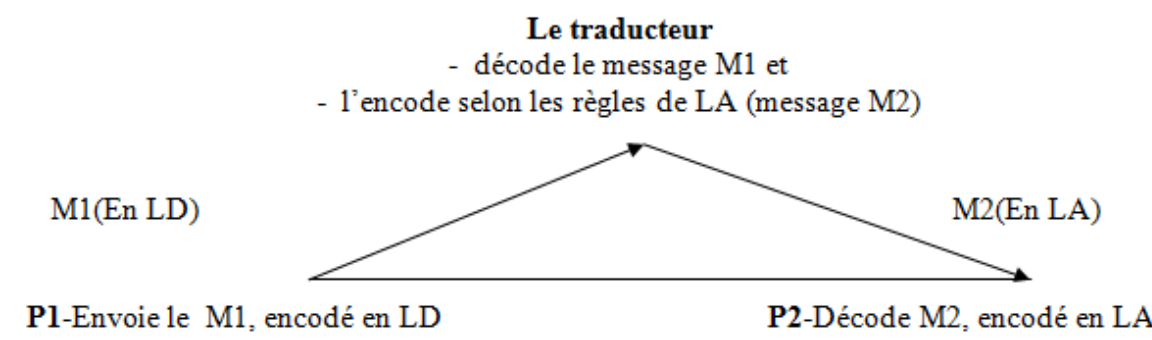

Schéma triangulaire du processus traduisant

3 Le processus traduisant n'est pas directement observable. Ce qui est accessible à l'observation, ce sont uniquement les produits issus de ce processus ainsi que, grâce au 
progrès relativement récent des neurosciences, certaines manifestations cliniques (activation de certaines aires cérébrales, par exemple) observables en situation d'expérimentation.

James S. Holmes, un important chercheur par ses études sur la traduction, a proposé une approche mentale pour modéliser le processus de traduction, communément appelé théorie « cartographique ».

D'après Holmes, le processus de traduction est un processus en plusieurs étapes; pendant que nous traduisons des phrases, nous avons une carte du texte original dans notre esprit et en même temps, une carte du genre de texte que nous voulons produire dans la langue cible. Même lorsque nous traduisons en série, nous avons ce concept de structure de sorte que chaque phrase de notre traduction est construite non seulement à partir de la phrase du texte original mais avec les deux cartes du texte original et du texte traduit lesquelles nous entretenons tout en exécutant la traduction. (Holmes $1988: 96$ )

\section{Déverbalisation}

6 La traduction n'est pas une pratique linéaire qui part d'un texte-source (Ts) pour arriver à un texte-cible (Tc). S'il est vrai que la traduction est un terme qui désigne parfois une activité professionnelle, d'autres fois un produit (le texte-cible), ce terme se rapporte aussi et surtout à un processus mental, binaire (Ladmiral 2005) ou en trois phases (selon Seleskovitch et Lederer 2001). Il s'agit d'une première phase (lecture-interprétation) et d'une phase seconde (recréation-cible), en passant par la déverbalisation, que les théoriciens de l'ESIT distinguent comme une troisième phase. Entre les deux phases du processus de transfert, il y a donc un tertium quid, faisant passer le message du niveau verbo-linguistique de la langue-source au niveau logico-cognitif. Ce passage entre la première et la deuxième phase est considéré comme un moment de grande tension psychologique où le traducteur met en jeu l'alchimie mentale d'une dialectique mémorielle délicate, aux termes de laquelle il lui faut à la fois oublier les signifiants de la langue-source, les laisser tomber, et n'en retenir que les signifiés ou, plus précisément le sens du message, pour le réincarner dans les signifiants à venir de la langue-cible (fig.2).

C'est le schéma du fameux «salto mortale» de la déverbalisation ladmiralienne : 
Figure 2
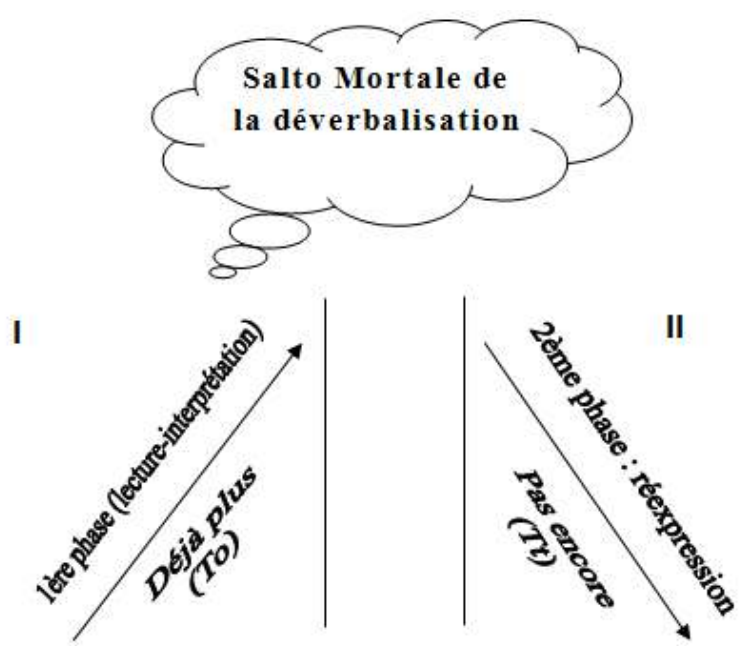

(To)

$(\mathrm{Tt})$

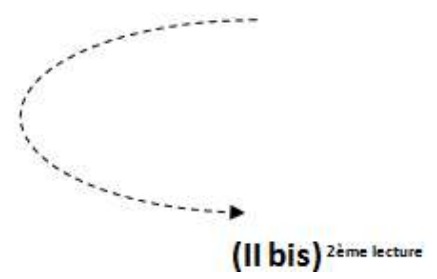

Salto mortale de la déverbalisation (Ladmiral, $2005: 1$ )

8 López Martínez (2005 : 39) a tenté d'expliquer comment s'opère la déverbalisation au niveau psychologique chez un interprète :

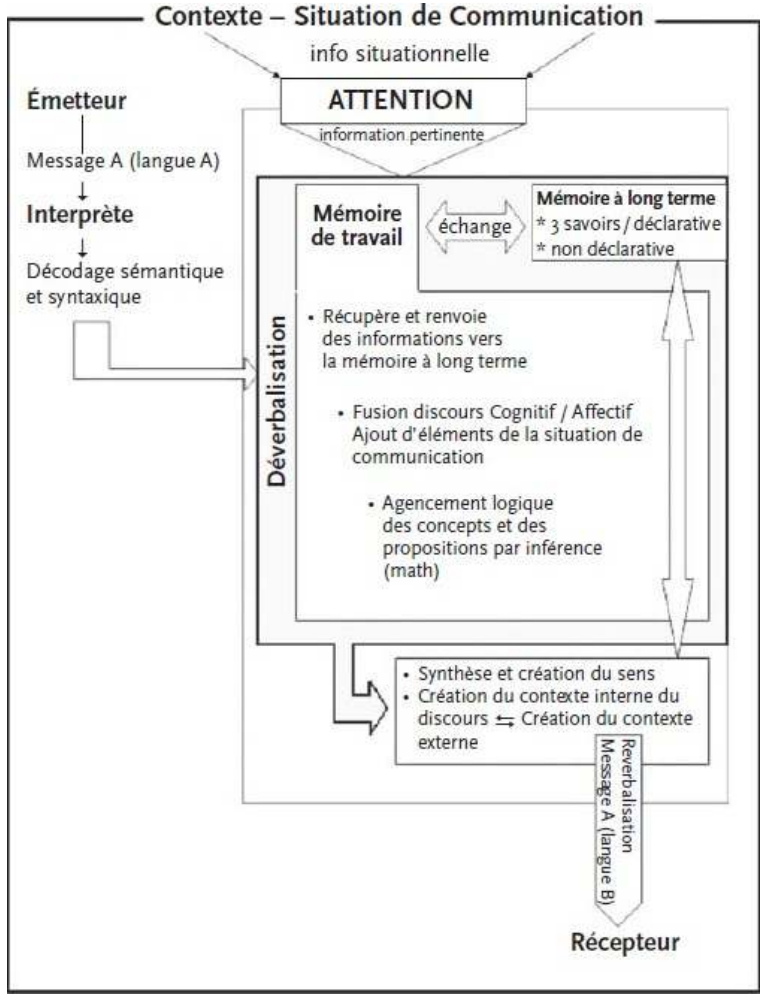


9 L'émetteur du discours va prononcer le message A (en langue A) qui va arriver aux oreilles de l'interprète. Ce dernier fera office de récepteur temporaire du message et va déverbaliser celui-ci, c'est-à-dire le désambigüiser par une alchimie dont paradoxalement - le bon interprète maitrise l'application sans en décrypter le processus (Balliu $2007:$ 10).

\section{Méthodologies}

Parmi les nombreuses méthodes expérimentales sur le processus de la traduction, on en distingue deux, la méthode de collection de données in vivo, c'est-à-dire prises sur le vif pendant l'opération traduisante, et la méthode de collection de données in vitro, c'est à dire après que le traducteur a eu fini de traduire.

\subsection{Méthode du raisonnement à voix haute ou TAP (in vivo)}

11 La méthode du raisonnement à voix haute (Think aloud protocol) sert à étudier les processus cognitifs qu'une personne doit mobiliser en cours d'action, encadrée par des protocoles (transcription, enregistrement, questionnaire, etc.). Cette méthode consiste à demander au traducteur de dire tout haut ce qu'il pense tout bas pendant qu'il traduit. Ses commentaires verbaux ainsi que son comportement non verbal sont enregistrés sur vidéo. Ces données spontanées sont par la suite transcrites en protocoles de verbalisation, appelés TAP dans la littérature traductologique.

12 L'utilisation de ce moyen logique est le plus souvent attribuée à Ericsson et Simon (expérimentation de 1983, 1984, 1993, 2 ééd.) et il a été depuis largement employé pour l'analyse du processus de résolution de problèmes. Ce n'est que vers la fin des années 80 , que la méthode TAP commence à être appliquée à la traduction professionnelle, Tirkkonen-Condit (1987), Jääskelaïnen (1987), Séguinot (1989), Lorscher (1991), Dancette \& Ménard (1996).

13 Löscher (1986 et 1991) a présenté une étude importante de TAP, dans laquelle 48 apprenants allemands de l'anglais comme langue étrangère produisent 52 traductions en anglais ou en allemand. Ils ont été invités à produire une traduction orale d'un texte écrit tout en pensant à haute voix et ne sont pas autorisés à utiliser les dictionnaires (cela visait à assurer un plus grand nombre de processus de résolution de problèmes présents dans les protocoles). Les transcriptions des séances ont ensuite été analysées et un certain nombre de stratégies de traduction ont été reconnues. Selon Löscher chaque stratégie est formée d'une série d'éléments de base qui peuvent être combinées de manières différentes. Un processus de traduction, à son tour, est formé d'une série de stratégies, qui peuvent également être combinées de manières différentes. La conclusion générale dégagée de cette étude est que TAP peut fournir des données fiables et utiles, à condition que l'analyste les interprète d'une façon systématique et méthodologiquement contrôlée.

\subsection{Translog (in vivo)}

Translog est un logiciel présenté par Jakobsen \& Schou (1999), qui a la possibilité d'enregistrer en temps réel, accéléré ou réduit toutes les activités d'écriture du sujet, 
correspondant aux touches du clavier de l'ordinateur sur lesquelles a appuyé le traducteur, aux mouvements de la souris reliée à son ordinateur ainsi qu'aux pauses entre les activités d'écriture.

Selon Jakobsen, "père » du logiciel Translog, les données Translog peuvent fournir le matériau brut aux études du processus traductionnel. ${ }^{1}$ La manière de se mouvoir du traducteur dans son texte reflète un comportement qui se prête particulièrement bien à l'observation, à partir duquel on peut tirer certaines régularités. Le comportement du traducteur s'observe en fonction:

- des lettres et des mots qu'il tape sur le clavier ainsi que des mouvements de la souris.

- des corrections qu'il apporte aux solutions provisoires retenues au fur et à mesure qu'il avance dans son texte.

- des révisions lors de relecture après avoir achevé la tâche.

- des pauses pendant l'opération traduisante, à savoir aussi bien la longueur des pauses initiale et finale, que toutes les autres pauses qui interviennent entre les mots ou à l'intérieur même d'un mot.

Le logiciel est disponible en deux différents types d'interface: les interfaces utilisateur et superviseur. L'interface utilisateur est envisagé au traducteur, et son écran est divisé en deux: l'écran du haut permet l'affichage d'un texte source, tandis que l'écran du bas est utilisé pour traduire le texte. Le logiciel enregistre toutes les actions du clavier et de la souris, et bien des fois la durée de l'activité de traduction (entre la pression sur le bouton de « go » et du « stop ») et les pauses entre toutes les actions et les mouvements.

Les données informatisées sont stockées dans un fichier qu'il est possible de faire apparaître sur l'écran en deux versions : play-back cinématique et graphique. Dans la version play-back cinématique, toutes les données enregistrées peuvent être affichées sur l'écran comme un film. Dans la version graphique, on peut visualiser non seulement les lettres correspondant aux touches sur lesquelles a appuyé le traducteur, mais aussi les symboles correspondant aux touches du clavier autres que celles qui renvoient aux lettres de l'alphabet ainsi qu'aux mouvements de la souris.

Symboles de Translog :

\begin{tabular}{|c|c|}
\hline * & unité de pause \\
\hline [*:n.n] & unité de pause étendue \\
\hline \& & touche interligne \\
\hline$\bullet$ & barre espace \\
\hline$\nabla \Phi$ & touche d'effacement à gauche ou à droite \\
\hline [Ctrl] & touche contrôle \\
\hline$\leftarrow \downarrow \uparrow \rightarrow$ & touches flèches \\
\hline [Shft] & touche majuscule \\
\hline
\end{tabular}

\subsection{Méthodes in vitro}

Les approches in vitro consistent en observations faites après la fin de la tâche :

- Réponses aux questionnaires.

On pose des questions à propos du texte afin de compléter nos données.

- Entretiens

- Commentaires et réflexions généré(e)s par la fonction play-back du logiciel Translog qui permet de visualiser l'opération traduisante en temps réel, accéléré ou réduit. 


\begin{tabular}{|l|l|l|l|l|}
\hline Répondant & Age & Sexe & Langues (sauf persan) & Expérience \\
\hline Farzaneh (Sujet 1) & 25 & Féminin & Français-anglais & 6 ans \\
\hline $\begin{array}{l}\text { Nasrin } \\
\text { (Sujet 2) }\end{array}$ & 28 & Féminin & Français-anglais & 8 ans \\
\hline $\begin{array}{l}\text { Hadi } \\
\text { (Sujet 3) }\end{array}$ & 26 & Masculin & Français-anglais-arabe & 7 ans \\
\hline $\begin{array}{l}\text { Vahid } \\
\text { (Sujet 4) }\end{array}$ & 28 & Masculin & Français- anglais & 6 ans \\
\hline $\begin{array}{l}\text { Ali } \\
\text { (Sujet 5) }\end{array}$ & 26 & Masculin & Français-anglais & 6 ans \\
\hline
\end{tabular}

\subsubsection{Texte à traduire}

Le texte original français qui a servi à l'expérience est un texte tiré du test de TCF en Iran pour l'évaluation de compréhension en français. Il a été sélectionné pour les raisons suivantes : c'est un texte original en français, qui contient quelques structures et des mots qui n'existent pas en persan, et cela exige un effort cognitif du traducteur. Ce n'est ni très difficile ni facile à traduire. Il y a des phrases courtes dans le premier paragraphe et des phrases longues dans le deuxième. Le texte ne comporte que 236 mots, avec une forme bien cohérente, donc ce n'est pas fatiguant pour les sujets.

"Génération kangourou"

Ils dorment dans leur lit d'enfant, mais ont entre 20 et 30 ans. Lovés dans le nid 
familial ... Selon une enquête de l'institut Louis Harris (novembre 1997), un jeune sur deux entre 21 et 24 ans, un sur cinq entre 25 et 29 ans reste au chaud chez ses parents.

Ils accumulent les diplômes mais collectionnent les petits boulots en CDD. Les emplois stables à temps complet sont devenus une denrée rare pour ceux qui n'ont pas d'expérience professionnelle. Dans ces conditions, il n'est pas étonnant que les 20 - 30 ans hésitent à quitter le toit familial.

Mais l'allongement des études, la précarité économique et le chômage ne suffisent pas à expliquer cette cohabitation prolongée des jeunes et de leurs parents. Ces derniers, en effet, ne sont pas mécontents de garder leurs enfants à la maison. Une manière, peut-être, pour cette génération de soixante-huitards, de retenir leur jeunesse. Et le risque est là : celui de maintenir les jeunes dans l'enfance et de les empêcher de grandir. Françoise Sand, conseillère familiale et conjugale, dénonce le danger psychologique qui pèse sur les pré-adultes et suggère aux parents de les pousser dehors, quitte à les assister financièrement pendant un certain temps. Et ne serait-ce pas là aider véritablement cette génération dont neuf jeunes sur dix, d'après le sondage Louis Harris, déclarent qu'ils quitteraient le foyer familial s'ils en avaient le choix?

\section{Indices d'efforts cognitifs} expérimentale The effects of time on cognitive processes and strategies in translation fondée sur les modèles de Bereiter et Scardamalia (1987), dits Knowledge telling et Knowledge transforming, qui servent à décoder des processus mentaux ayant trait à la production unilingue de textes, Jensen $(2000)^{2}$ a essayé d'identifier un certain nombre d'indices susceptibles de refléter les efforts cognitifs du traducteur :

26

Elle a présenté les sept indices suivants :

1. pauses

2. reports de décision

3. corrections

4. révisions

5. consultation de dictionnaires

6. remarques sur les problèmes rencontrés

7. remarques sur les objectifs fixés

27 Ces paramètres ont été sélectionnés par Jensen dans la perspective d'une analyse statistique. En ce qui concerne les corrections, elles sont divisées en deux catégories: les corrections dans la phase d'écriture et les corrections dans la phase de révision. Dans notre étude, nous avons combiné les trois derniers indices dans l'indice de « consultation de dictionnaires ", donc nous adoptons les cinq premiers indices. C'est parce qu'on n'a pas enregistré les commentaires des sujets traduisant lors de la tâche pour ne pas les déconcentrer. D'ailleurs, grâce au questionnaire, nous avons accès aux informations complémentaires. 


\section{Manifestation des indices}

\subsection{Pauses}

Les pauses du traducteur nous signalent un effort cognitif. Une étude sur des pauses de Joost Schilperoord (1996) présente l'idée selon laquelle les pauses sont révélatrices d'activités mentales : «the time interval between the presentation of a stimulus and the production of a response is taken to be a measure of the amount of cognitive processes the subject needs for his response » (Schilperoord $1996: 18$ cité par Jensen $2000: 101$ ) Plus les pauses sont longues, plus l'effort cognitif fourni pour résoudre le problème en question semble soutenu. "The more the delays (pause time J.S.) the more cognitive operations (e.g. processing effort, J.S.) are required by the output »(Op. cite: 59).

D'après Jakobsen, les pauses de plus de 10 secondes sont les signes d'un effort cognitif particulier ${ }^{3}$. C'est donc cette durée de pause - un minimum de 10 secondes - que nous prenons ici comme indice d'un effort cognitif particulier.

Le tableau ci-dessous nous montre les pauses marquées par les sujets.

\begin{tabular}{|l|l|l|l|l|l|l|}
\hline Pauses & $\begin{array}{l}\text { Pauses 10s à } \\
20 \text { s }\end{array}$ & $\begin{array}{l}\text { Pauses 20s à } \\
30 \text { s }\end{array}$ & $\begin{array}{l}\text { Pauses plus de } \\
30-60 \text { s }\end{array}$ & $\begin{array}{l}\text { Pauses plus } \\
\text { de 60s }\end{array}$ & $\begin{array}{l}\text { Nombre } \\
\text { Totale }\end{array}$ & $\begin{array}{l}\text { Durée } \\
\text { totale }\end{array}$ \\
\hline Sujet 1 & 18 & 1 & 1 & 1 & 21 & $21: 54$ \\
\hline Sujet 2 & 9 & 7 & 2 & 0 & 18 & $19: 05$ \\
\hline Sujet 3 & 9 & 3 & 0 & 0 & 12 & $17: 52$ \\
\hline Sujet 4 & 9 & 4 & 2 & 0 & 15 & $18: 58$ \\
\hline Sujet 5 & 13 & 4 & 2 & 0 & 19 & $24: 48$ \\
\hline
\end{tabular}

La plus longue pause a été enregistrée pour le sujet 01, et c'était dans la phase de révision.

\subsection{Report de décision}

Le report de décisions est aussi l'indice d'une activité cognitive. Le traducteur peut choisir de repousser à plus tard sa solution à un problème et passer à un autre segment du texte. Dans les données Translog, ces décisions se reconnaissent aux symboles :

\section{सैझ}

ou parfois par les points de suspension : (...).

Dans notre donnée de Translog, nous avons juste trouvé 4 fois cet indice. Deux fois pour le Sujet 4, deux fois pour le Sujet 5 . Tous les deux avaient choisi de reporter la traduction du titre à plus tard.

Les données de translog du Sujet 4 :

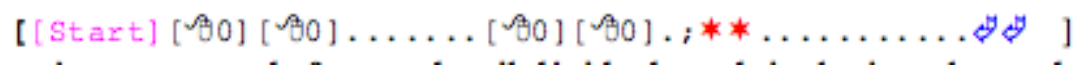


Sujet 5 , après une pause de 9 secondes, il décide de traduire le titre plus tard :

[*в凶********........

7 Dans le texte traduit par le sujet 4, on y voit les points de suspension. Ces points de suspension indiquent que la solution au problème de reformulation est reportée à plus tard. Il replace ces points après avoir fini le paragraphe :

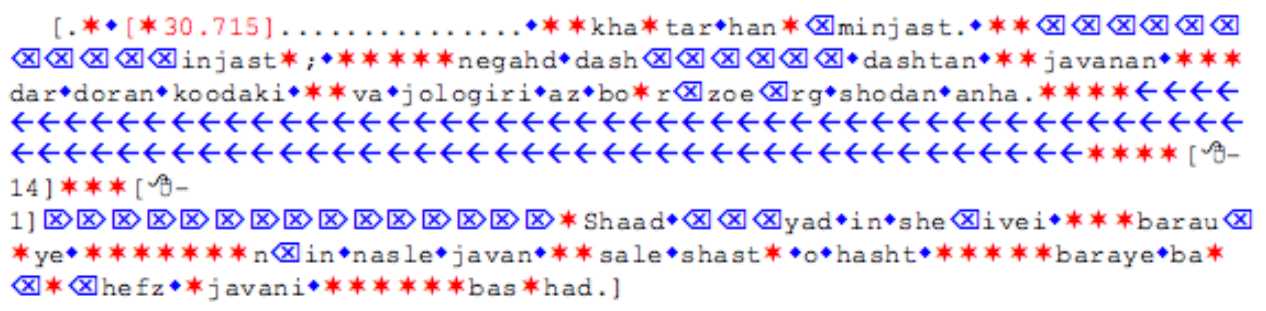

Comme on le voit, la traduction de la phrase «Une manière, peut-être, pour cette génération de soixante-huitards, de retenir leur jeunesse » a été reportée à plus tard.

Le sujet 5 , a fait également un report de décision pour la traduction de "dénonce le danger psychologique qui pèse sur les pré-adultes ». Selon ses notes, vu que deux verbes « dénonce » et «suggère » se succèdent, il décide d'abord de traduire la phrase la plus longue.

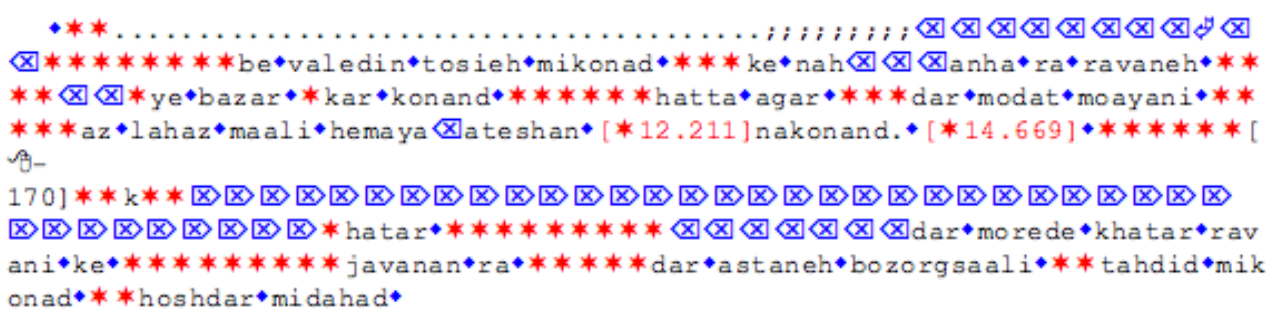

Nous n'avons pas trouvé dans les données de Translog d'autres reports de décision. Dans le tableau ci-après, il s'agit du nombre de cet indice :

\begin{tabular}{|l|l|}
\hline & Report de décision \\
\hline Sujet 1 & 0 \\
\hline Sujet 2 & 0 \\
\hline Sujet 3 & 0 \\
\hline Sujet 4 & 2 \\
\hline Sujet 5 & 2 \\
\hline
\end{tabular}

\subsection{Corrections}

40 Le traducteur se corrige au fur et à mesure qu'il avance dans son texte. Ce sont les corrections. Elles se reconnaissent dans les données Translog aux mouvements d'effacement en arrière signalés par le symbole : 
ou la touche:

\section{$\leftarrow$}

Translog enregistre toutes les éliminations faites par le traducteur, mais sans identifier les distinctions. Les corrections des erreurs de frappe ne sont donc pas prises en considération comme une charge cognitive mais sont enregistrées dans le logiciel.

Nous allons donner quelques exemples des corrections faites par nos sujets qui les obligent à opérer une activité mentale.

Exemples :

Le sujet 1 a fait deux fois la correction pour traduire l'expression «quitte à » dans la phrase suivante :

« ...quitte à les assister financièrement pendant un certain temps »

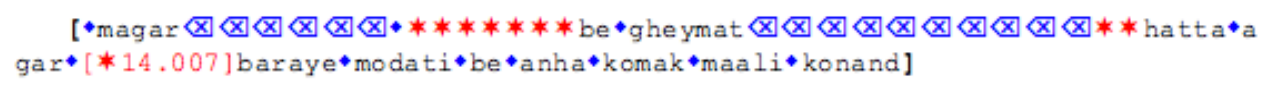

Premièrement il choisit le mot "[magar] مكر " (sauf que), puis il essaie l'expression persane de "به قيمت [be qeymat]» (au prix de) et enfin il décide d'y mettre le terme «[même si] حتى فيمات (Même si).

Un autre exemple est la correction de la phrase ci-dessous par le Sujet 2 :

« Dans ces conditions, il n'est pas étonnant que les 20 - 30 ans hésitent à quitter le toit familial. »

Pour traduire l'expression « il n'est pas étonnant que », le Sujet 2 utilise tout d'abord une modulation "[tabiye ast ke] طبيعى است كه" (c'est normal que mais après une pause de 12 secondes environ, il corrige en la traduisant littéralement «[tajobi nadarad] تعجبى ندارد《. (Ce n'est pas étonnant que).

[dar*chenin*sharyeti***,**tabi*ast*ke*****é凶20*saleh*ha*jorat**** *nadarand [*12.313] khaneye pedari ra*tark konand.* $* \leftarrow \leftarrow \leftarrow \leftarrow \leftarrow \leftarrow \leftarrow \leftarrow \leftarrow \leftarrow \leftarrow \leftarrow$

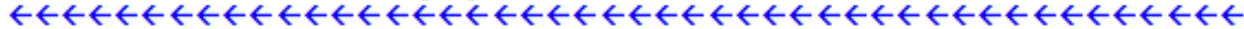
$\leftarrow \leftarrow \leftarrow \leftarrow \leftarrow \leftarrow * \otimes \otimes \otimes \otimes * * * * * \rightarrow \rightarrow \rightarrow \rightarrow \otimes \otimes \otimes \otimes t a j a b i * *$ nadarad*]

Le Sujet 3 traduit très vite dans le mode de mot à mot, l'expression « Pendant un certain temps» «[dar yek modat khass] در يك مدت خاص», mais il la corrige en choisissant une

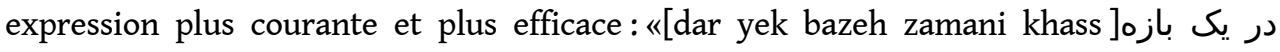
زمانى خاص"

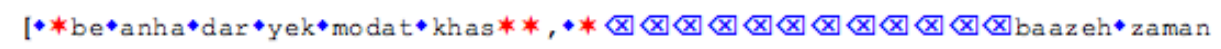
$i * k h a \otimes \otimes * 1$

Le Sujet 5 a effectué le plus grand nombre de corrections parmi nos sujets. Comme tous les sujets, Sujet 5 rencontre des difficultés pour la traduction des verbes conditionnels. Les données Translog nous signalent plusieurs corrections dans le dernier paragraphe du texte : 
[]区*****[\$46] [*12.561] Tebgh nazar*anji haris**********az*har**1

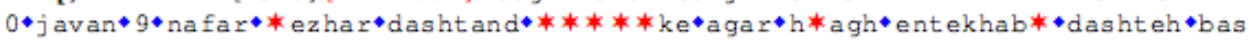

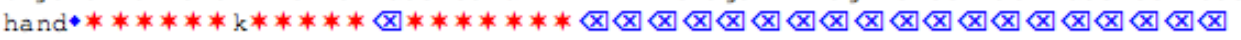

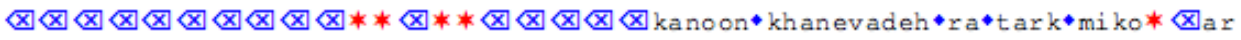
dand aga* $\otimes \otimes \otimes \otimes \otimes \otimes \otimes \otimes \otimes \otimes \otimes k o n a n d * a g a r * h a g h e * e n t e k h a b * d a s * h t a n d . * * * *$

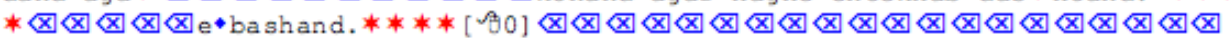

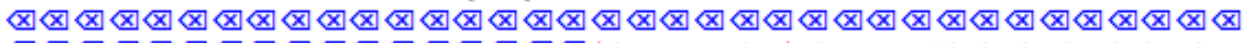

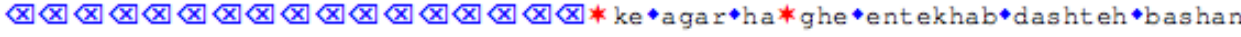

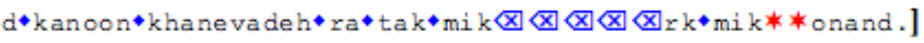

Le tableau ci-après est récapitulatif du nombre de corrections totales:

\subsection{Révisions}

\section{Le Sujet 1 :}

Les corrections apportées au texte après la fin de la phase d'écriture ou parfois après la fin du paragraphe nous indiquent une activité réflexive et mentale.

Presque tous nos répondants ont fait la phase de révision, quelques uns très rapidement, quelques autres plus lentement et avec attention. Certains d'entre eux élaborent aussi de nouvelles corrections à cette phase.

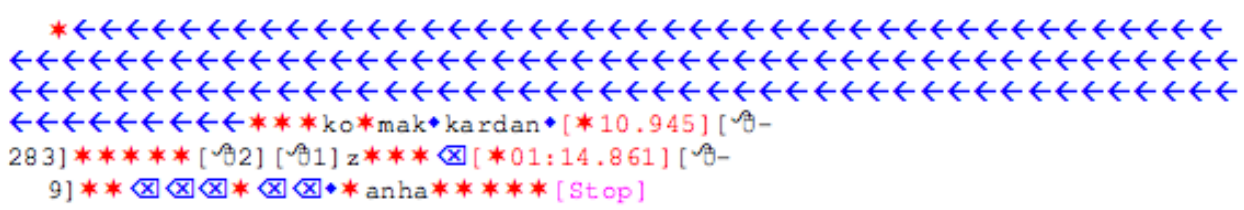

Il a fait deux modifications dans la phase de correction. Premièrement, il fait un retour au dernier paragraphe et ajoute le verbe "kikomak kardan : aider] au début de la

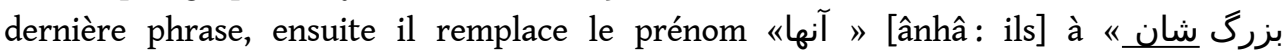
شدن «shân] à la ligne 12]

Il a fait deux pauses importantes dans cette phase, l'une de 10 secondes et l'autre est de $[10: 14]$.

51 Le sujet 2:

[* *** * $\leftarrow \rightarrow \rightarrow \rightarrow \rightarrow \rightarrow \rightarrow \rightarrow \rightarrow \rightarrow \rightarrow \rightarrow \rightarrow \rightarrow \rightarrow \rightarrow \rightarrow \rightarrow \rightarrow \rightarrow \rightarrow \rightarrow \rightarrow \rightarrow \rightarrow \rightarrow \rightarrow \rightarrow \rightarrow \rightarrow \rightarrow \rightarrow \rightarrow \rightarrow \rightarrow \rightarrow \rightarrow \rightarrow \rightarrow \rightarrow$ $\rightarrow \rightarrow \rightarrow \rightarrow \rightarrow \rightarrow \rightarrow \rightarrow \rightarrow \rightarrow \rightarrow \rightarrow \rightarrow \rightarrow \rightarrow \rightarrow \rightarrow \rightarrow \rightarrow \rightarrow \rightarrow \rightarrow \rightarrow \rightarrow \rightarrow \leftarrow \rightarrow \rightarrow \rightarrow \rightarrow \rightarrow \rightarrow \rightarrow \rightarrow \rightarrow \rightarrow \rightarrow \rightarrow \rightarrow \rightarrow \rightarrow \rightarrow \rightarrow \rightarrow \rightarrow \rightarrow$ $\rightarrow \rightarrow \rightarrow \rightarrow \rightarrow \leftarrow \leftarrow \leftarrow \leftarrow \leftarrow \leftarrow * * * *$ [ [

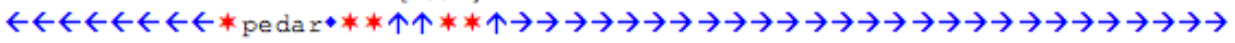
$\rightarrow \rightarrow \rightarrow \rightarrow \rightarrow \rightarrow \rightarrow \rightarrow \rightarrow \rightarrow \rightarrow \rightarrow \rightarrow \rightarrow \rightarrow \rightarrow \rightarrow \rightarrow \rightarrow \rightarrow \rightarrow \rightarrow \rightarrow \rightarrow \rightarrow \rightarrow \rightarrow \rightarrow \rightarrow \rightarrow \rightarrow \rightarrow \rightarrow \rightarrow \rightarrow \rightarrow \rightarrow \rightarrow \rightarrow \rightarrow \rightarrow \rightarrow \rightarrow \rightarrow \rightarrow$ $\rightarrow \rightarrow \rightarrow \leftarrow * * * * * * *$ pish*az*boloogh*[*14.272] [Stop]]

Le Sujet 2 a fait juste deux ajouts dans la phase de révision. En lisant sa traduction, il se rend compte qu'il n'a pas traduit l'adjectif «familiale» dans la dernière phrase, donc il ajoute le mot " يدر ».[pedar : père]

Après avoir fait 52 mouvements de souris, un retour à la ligne de 13 du texte, il cherche à trouver un terme pour bien présenter le sens de " pré-adulte », il décide d'ajouter ou bien d'étoffer en écrivant : يڤيش از بلوغ ». [pish az boluq : pré-adulte].

Le Sujet 3 :

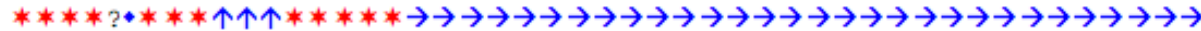

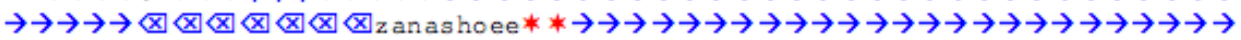
$\rightarrow \rightarrow \rightarrow \rightarrow \rightarrow \rightarrow \leftarrow \leftarrow \leftarrow \leftarrow \leftarrow \leftarrow \leftarrow \leftarrow \leftarrow \leftarrow * * * \mathrm{kh} * \mathrm{t} \otimes \otimes *$ [अ339]********[Stop] 
Il n'a pas effectué de grands changements dans la phase de révision. Il retourne aux adjectifs "familiale et conjugale " en ajoutant le mot "زناشويى" et ensuite pour la traduction «psychologique» il remplace l'adjectif "روانشناسى " [ravânshenâsi : psychologie] » par "روانشناختى[ravânshenâkhti : psychanalyse ]».

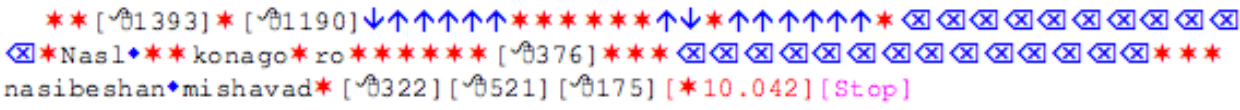

Après avoir achevé la traduction, il revient tout de suite au titre pour le traduire. Pendant la phase de révision, il décide de changer sa traduction du verbe "collectionner » à la quatrième ligne. Il choisit le verbe "[nasibeshân mishavad]دنصيبشان مى شود " ce qui rend bien le sens par rapport au verbe "بيدا مى كنند[peyda mikonand : ils trouvent] ». Le nombre des mouvements de (mouse) souris est également significatif.

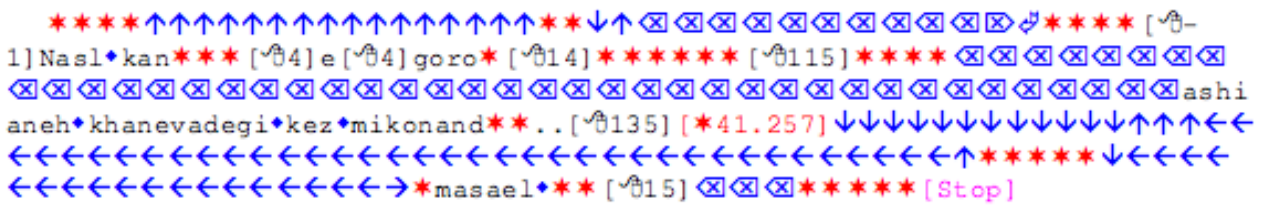

Dans les données de Translog du Sujet 5, trois corrections ont été enregistrées. Comme le sujet 4 , il a reporté la traduction du titre à la fin, il traduit le titre, puis il corrige la traduction de l'expression " dans le nid familial ». Il préfère l'expression persane «[kez

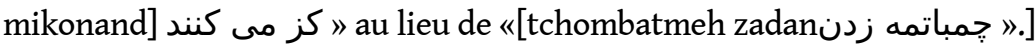

Le tableau ci-après nous indique les révisons faites par nos sujets :

\section{Recours au dictionnaire}

La consultation des dictionnaires bilingues et unilingues, ou des fiches terminologiques ou toutes autres sources d'informations électroniques dans la phase de reformulation nous signale les efforts cognitifs du traducteur. Le sujet traduisant cherche en général dans le dictionnaire un appui, moins pour trouver le mot ou l'expression correspondant, que pour se sortir d'une impasse. Il peut arriver, parfois, qu'un dictionnaire bilingue soit insuffisant pour trouver l'équivalence exacte du mot et il faut consulter le dictionnaire unilingue en vue de bien lire les explications et de comprendre la signification.

Consulter un dictionnaire n'est pas toujours pour connaître le sens du mot mais la plupart du temps c'est pour trouver les différents équivalents proposés et en choisir un concernant les aspects culturels et esthétiques.

Afin d'avoir accès aux données concernant les utilisations de dictionnaire chez nos sujets traduisant, nous leur avons demandé de les noter sur la page du texte de départ. En plus, dans le questionnaire on leur demande quelques explications sur ce sujet, par exemple les noms des dictionnaires utilisés ou les raisons de recours à ceux-ci.

Selon les notes et les commentaires des sujets traduisant, nous avons divisé les motifs de consulter le dictionnaire en trois catégories. La première, concerne les mots dont le traducteur ne connaît pas le sens et il le cherche pour bien le comprendre. Dans la deuxième catégorie, il comprend le sens mais il ne sait pas l'équivalent dans la langue d'arrivée. La troisième catégorie, le sujet traduisant connaît le sens, et il le comprend 
bien, mais cependant il est à la recherche d'autres propositions dans le dictionnaire ou pour s'assurer de l'équivalent choisi.

Le tableau ci-dessous est le récapitulatif des recours au dictionnaire chez les cinq sujets d'étudiants :

\begin{tabular}{|c|c|c|c|c|}
\hline $\begin{array}{l}\text { Recours au } \\
\text { dictionnaire }\end{array}$ & $\begin{array}{l}\text { Je ne } \\
\text { connais pas } \\
\text { le sens }\end{array}$ & $\begin{array}{lr}\text { Je comprends le } \\
\text { sens mais je ne } \\
\text { connais } & \text { pas } \\
\text { l'équivalent } & \text { en } \\
\text { persan } & \end{array}$ & $\begin{array}{l}\text { Je connais bien le sens et } \\
\text { l'équivalent, je veux } \\
\text { consulter d'autres } \\
\text { équivalents ou m'assurer } \\
\text { de mon choix }\end{array}$ & $\begin{array}{l}\text { Nombre total de } \\
\text { recours au } \\
\text { dictionnaire }\end{array}$ \\
\hline Sujet 1 & $\begin{array}{l}\text { Lové, } \\
\text { Denrée }\end{array}$ & $\begin{array}{l}\text { CDD, quitte à, rester } \\
\text { au raud, } \\
\text { cohabitation }\end{array}$ & $\begin{array}{l}\text { accumuler, boulot, foyer, } \\
\text { énoncer }\end{array}$ & 10 \\
\hline Sujet 2 & $\begin{array}{l}\text { Lové, quitte } \\
\text { à }\end{array}$ & $\begin{array}{l}\text { CDD, } \\
\text { soixante-huitards }\end{array}$ & $\begin{array}{l}\text { Nid familial, dénoncer, } \\
\text { précarité, denrée rare, } \\
\text { enquête }\end{array}$ & 7 \\
\hline Sujet 3 & $\begin{array}{l}\text { Quitte à, } \\
\text { précarité, } \\
\text { lové }\end{array}$ & $\begin{array}{l}\text { CDD, cohabitation, } \\
\text { nid familial, rester } \\
\text { au chaud }\end{array}$ & $\begin{array}{l}\text { Collectionner, conjugale, } \\
\text { suggère, boulot }\end{array}$ & 11 \\
\hline Sujet 4 & $\begin{array}{l}\text { Lové, } \\
\text { denrée rare }\end{array}$ & CDD, quitte à & 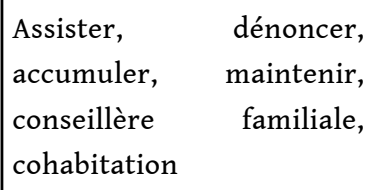 & 10 \\
\hline Sujet 5 & $\begin{array}{l}\text { Lové, } \\
\text { denrée } \\
\text { rare, CDD }\end{array}$ & $\begin{array}{l}\mathrm{CDD}, \quad \text { conseillère } \\
\text { familiale, soixante- } \\
\text { huitards }\end{array}$ & $\begin{array}{l}\text { Suggérer, boulots, quitte } \\
\text { à, nid familial }\end{array}$ & 12 \\
\hline
\end{tabular}

Deux termes « CDD » et «lové » sont le cherchés dans le dictionnaire par tous les sujets.

\section{Conclusion}

Le processus traductionnel est une activité intellectuelle complexe de résolution de problèmes. Pour déterminer les cas où le traducteur fait preuve d'un effort cognitif soutenu pour résoudre un problème de traduction, nous avons fait correspondre un certain nombre d'indices aux efforts cognitifs présumés du traducteur. Il semble logique de penser que plus les indices sont nombreux, plus la charge cognitive du traducteur est lourde. C'est dans cette perspective que nous proposons de représenter quantitativement la charge cognitive du traducteur selon les statistiques du Translog et des notes du traducteur.

\begin{tabular}{|l|l|l|l|l|l|}
\hline Charge cognitive & Sujet 1 & Sujet 2 & Sujet 3 & Sujet 4 & Sujet 5 \\
\hline
\end{tabular}




\begin{tabular}{|l|l|l|l|l|l|}
\hline Indice 1 & 21 & 18 & 12 & 15 & 19 \\
\hline Indice 2 & 0 & 0 & 0 & 2 & 2 \\
\hline Indice 3 & 197 & 124 & 279 & 216 & 362 \\
\hline Indice 4 & 3 & 2 & 2 & 2 & 3 \\
\hline Indice 5 & 10 & 7 & 11 & 10 & 12 \\
\hline Totale & 231 & 151 & 304 & 245 & 398 \\
\hline
\end{tabular}

63 Comme le tableau ci-dessus nous le signale, du point de vue quantitatif, le sujet 5 a accompli plus d'efforts cognitifs que les autres.

Grâce aux indices d'efforts cognitifs, nous pourrions bien accéder aux problèmes des traductions français-persan et connaître les tournures grammaticales, syntaxiques et culturelles qui bloquent le traducteur iranien. Le résultat de ces problèmes, comme un diagnostic, nous servira dans l'enseignement de la traduction. Par exemple, si on veut voir quels sont les problèmes de compréhension liés à une connaissance insuffisante de la culture française, nous pourrions envisager comme méthode de sélectionner un certain nombre de discours français faisant allusion à des éléments culturels français, enregistrer les traducteurs et voir les fautes et maladresses, puis les interroger (questionnaire) pour avoir davantage d'informations sur ce qu'ils ont compris ou pas compris et pourquoi ils ont réagi comme ils ont réagi.

Cette étude sur l'activité traduisante s'était fixé l'objectif limité de décrire et de mesurer l'effort cognitif du traducteur. Les approches TAPs et Translog utilisées pour décrire l'activité cognitive du traducteur représentent une voie de recherche sur le processus de la traduction. L'analyse descriptive à laquelle j'ai procédé n'est qu'un tout premier pas en ce sens, surtout dans la combinaison linguistique française-persane.

\section{BIBLIOGRAPHIE}

Dancette J., (1992), « Des processus de traduction concomitants : compréhension et recherche d'équivalents ", ACLA, Vol 14, $\mathrm{N}^{\circ} 1$.

Dancette J., (1997), "Mapping meaning and comprehension in translation. Theoretical and experimental issues", in Joseph H. Danks, Gregory M. Shreve, Stephen B. Fountain et Michael K. McBeath (éds), Cognitive processes in translation and interpreting (p. 77-103). Thousand Oaks, CA, Sage.

Gile D., (2005), La traduction, la comprendre, l'apprendre, Paris, PUF.

Gile D., (1990), « La traduction et l'interprétation comme révélateur des mécanismes de production et de compréhension du discours ", Méta, Vol 35, $\mathrm{N}^{\circ} 1$. 
Holmes J. S., (1988), Translated! Papers on Literary Translation and Translation Studies, Amsterdam, Rodopi, ISBN 90-6203-739-9.

Jakobsen, Lykke Arnt \& Schou, Lasse, (1999), “Translog documentation” in G. Hansen (ed.) (1999) Probing the process in translation: methods and results, Copenhagen: Copenhagen Business School, 73-101.

Jakobsen, Lykke Arnt (2000), "Understanding the Process of Translation: The Contribution of Time-Delay Studies", in B. Englund Dimitrova (ed.) (2000), Översättning och tolkning, Stockholm: ASLAs skriftserie 12, 155-172.

Jakobsen, Lykke Arnt (2003), “Translation drafting by professional translators and by translation students", à paraître dans Traducción \& Communicación.

Jensen, Astrid (2000), The Effects of Time on Cognitive Processes and Strategies in Translation, PhD thesis, Copenhagen Business School.

Jääskelaïnen, Riitta (1987), What happens in a Translation Process: Think-aloud Protocols of Translation, Joensuu: University of Joensuu, Savonlinna School of Translation Studies.

Jääskelaïnen, Ritta (2002), “Think-aloud Protocol Studies into translation: an annotated bibliography”, Target 14 (1): 107-136.

Ladmiral J-R., (2005), Le « salto mortale de la déverbalisation », Meta, L, 2.

Lavaur, J. (1994), Traitement du texte et transfert interlinguistique. Approche psycho-cognitive de la compréhension et de la mémorisation de textes en langue maternelle et en langue étrangère, Université de Nice, Thèse de doctorat.

Martin B. et Lergos D., (2008), Psycholinguistique cognitive : lecture, compréhension et production du texte, Bruxelles, de Boeck.

López Martínez, M. (2005), Réflexions sur la déverbalisation, mémoire de fin d'études présenté à l'ISTI (Bruxelles) sous la direction de Chr. Balliu, non publié.

Lörscher W., (1991), Translation performance, translation process and translation strategies: A psycholinguistic investigation, Tübingen, Narr.

Lörscher W., (1992), "Process-oriented research into translation and implication for translation teaching”, Traduction, terminologie et rédaction, vol 5, n 1 . TTR.

Paula A. et Séguinot C., (2005), "Shortcuts, Strategies and General Patterns in a Process Study of Nine Professionals”, Journal des traducteurs / Meta: Translators' Journal, vol. 50, n² 2, 2005, p. 522-547.

Ronald J.A. et Thibault J.P., (1987), Problèmes de psycholinguistique, Bruxelles, Pierre Mardaga éditeur.

Rydning, Antin Fougner (2001), “The synecdochial device applied to translation seen against the conceptual" (2002) «Concept métaphorique et expression métaphorique dans une perspective cognitiviste », Dørum, H. (ed.) (2002) Romansk Forum 16, 723-733. http://www.digbib.uio.no/ roman/page21.html

Sabah G., (1989), L'intelligence artificielle et le langage, processus de compréhension, Paris, Hermès. Séguinot, Candace (1989), The Translation Process, Toronto, H.G. Publications.

Seleskovitch, D. et Lederer M., (2001), Interpréter pour traduire, Paris, Didier Érudition. 


\section{NOTES}

1. The central assumption is that a correlation exists between observable text production behaviour, including time delay 'behaviour', and the cognitive processes involved in text production; that it is possible to formulate hypotheses about translation acts from observations of translation behaviour. » (Jakobsen $2000: 158$ )

2. [...] as mental processes are not directly observable by the researcher, they need somehow to be inferred from a translator's behaviour. Thus, behaviour may act as clues to mental processes, and it was necessary for me to find ways in which I could identify indicators pointing to translation processes. (Jensen $2000: 97$ )

3. « With most translators, and in most situations, a time value greater than ten seconds will tend to identify text-initial and text-final delays, delays between paragraphs, and delays appearing less systematically before text segments that are particularly difficult to translate.» (Jakobsen $2000: 167)$

\section{RÉSUMÉS}

La traduction n'est pas une pratique linéaire qui part d'un texte-source (Ts) pour arriver à un texte-cible (Tc). La traduction est un exercice de compréhension et de ré-expression d'un discours qui suppose la mobilisation de connaissances linguistiques et thématiques. La progression des sciences cognitives au cours des vingt dernières années a permis de nourrir une réflexion multidisciplinaire aux frontières des neurosciences, de la psychologie, de la philosophie, de la linguistique et de l'intelligence artificielle. Dans cette recherche, en appliquant deux méthodologies Translog et TAPs (Think-aloud-protocols), nous essayons de découvrir la boite noire du traducteur et sa charge cognitive lors de l'acte de traduction français-persan.

Translation is not a linear act starting from a source text to reach the final text. The steps of the translation process (comprehension of the original text, cultural and linguistic mediation, shaping the target text) are not considered as a simple sequence of decoding and recoding messages. The progress of cognitive science in the last twenty years has helped feed a multidisciplinary study at the frontiers of neuroscience, psychology, philosophy, linguistics and artificial intelligence. In this research, using a combination of the two process-oriented approaches to study professional translation online: verbal reporting, better known as TAPs (think- aloud protocols), and Translog program for logging keyboard activity, we will examine the cognitive effort of translator French-Persian.

\section{INDEX}

Mots-clés : processus de traduction, traductologie cognitive, charge cognitive, Translog, ThinkAloud-Protocol, traduction français-persan

Keywords : translation process, Translation Studies, cognitive effort, Think- Aloud-Protocol, French-Persian translation 
AUTEUR

ESMAEEL FARNOUD

Laboratoire Modyco, Université Paris Ouest-Nanterre 